\title{
Testability and Epistemic Shifts in Modern Cosmology*
}

\author{
Helge Kragh \\ Centre for Science Studies, Aarhus University, 8000 Aarhus, Denmark. \\ E-mail address: helge.kragh@ivs.au.dk
}

\begin{abstract}
During the last decade new developments in theoretical and speculative cosmology have reopened the old discussion of cosmology's scientific status and the more general question of the demarcation between science and non-science. The multiverse hypothesis, in particular, is central to this discussion and controversial because it seems to disagree with methodological and epistemic standards traditionally accepted in the physical sciences. But what are these standards and how sacrosanct are they? Does anthropic multiverse cosmology rest on evaluation criteria that conflict with and go beyond those ordinarily accepted, so that it constitutes an "epistemic shift" in fundamental physics? The paper offers a brief characterization of the modern multiverse and also refers to a few earlier attempts to introduce epistemic shifts in the science of the universe. It further discusses the several meanings of testability, addresses the question of falsifiability as a sine qua non for a theory being scientific, and briefly compares the situation in cosmology with the one in systematic biology. Multiverse theory is not generally falsifiable, which has led to proposals from some physicists to overrule not only Popperian standards but also other evaluation criteria of a philosophical nature. However, this is hardly possible and nor is it possible to get rid of explicit philosophical considerations in some other aspects of cosmological research, however advanced it becomes.
\end{abstract}

Keywords: cosmology; multiverse; theory evaluation; testability; falsifiability; demarcation criteria; Popper

\footnotetext{
* Slightly revised preprint of paper submitted to Studies in History and Philosophy of Modern Physics.
} 


\section{Epistemic shifts and theory choice}

"Do we need to change the definition of science?" asked an article in the 7 May 2008 issue of New Scientist (Matthews, 2008). The occasion for the question was the recent appearance of a class of cosmological theories postulating the existence of an immense number of universes - a multiverse - rather than the single and unique universe in which we live. Multiverse physics or cosmology does not agree very well with the standard "definition" (or intuition) of science, which in this case was taken to include as a crucial element Popper's falsifiability criterion. The answer of some advocates of the multiverse has been to question or disregard the alleged definition of science and to look for a different understanding of what characterizes science, one that will allow multiverse physics to remain safely within the borders of science. They propose what I shall call an epistemic shift. ${ }^{1}$

As I shall use the term (Kragh, 2011), epistemic shifts refer to suggestions that traditional criteria of evaluation of scientific theories (or of theories claimed to be scientific) are no longer adequate and should therefore be replaced by new criteria that better fit the problems under investigation. In so far that they relate to the very criteria of what constitutes science, the suggested changes may in effect imply a new meaning or definition of what counts as science. They are, and are meant to be, changes in the demarcation between science and non-science. Such epistemic shifts are related to the paradigm shifts associated with

\footnotetext{
1 The term "epistemic shift" is occasionally used in sociological, political and literary theory, sometimes with a meaning close to Kuhnian paradigm shifts. In Michel Foucault's structuralist "archaeology" of knowledge, the emergence of political economy in the eighteenth century is said to have been an epistemic shift. Again, scholars have characterized the modern recognition of a global community with sustainable development as an epistemic shift in theories of international politics.
} 
revolutions in Kuhn's philosophy of science, but they differ from them in some respects. According to Kuhn's original view of 1962, methods of a research field, including values and rules of theory evaluation, are implicitly defined by the paradigm. He nonetheless argued for some timeless elements in science, one of them being that acceptability of theories is strongly regulated by observation and experiment. Whereas two competing paradigms are incommensurable, this is not the case with competing epistemic standards, which mostly differ in the ways they evaluate a theory. Given a new theory, which reasons do we have to believe in it or take it seriously? On the other hand, an epistemic shift may be so deep that it affects the significance of empirical tests, which is generally considered a stable epistemic virtue across paradigmatic shifts.

The question raised in New Scientist presupposes that there is a generally accepted and more or less invariant definition of science, a presupposition most philosophers, sociologists and historians of science will probably deny. All the same, and restricting myself to the physical sciences, there are undoubtedly some criteria of science and theory choice that the overwhelming majority of scientists agree upon and have accepted for at least two centuries. In a lecture of 1973, Kuhn (1977, pp. 320-339) suggested five "standard criteria for evaluating the adequacy of a theory," namely the following: (1) accuracy; (2) consistency, internal as well as external; (3) broadness in scope; (4) simplicity; (5) fruitfulness. The first criterion related to the theory's empirical power: within its domain, there must be "consequences deducible from a theory [that] should be in demonstrated agreement with the results of existing experiments and observations." Notice that Kuhn did not specifically refer to predictions, except that he included them under the notion of "fruitfulness," and that he apparently had confirmation rather than disconfirmation in mind. 
Kuhn (1977, pp. 290-291) was aware that the criteria or values may contradict each other in a concrete situation and that a relative weighing may therefore be needed; but such weighing cannot be unique, and so the system cannot fully determine an evaluation in a concrete case. In the context of modern cosmology Kuhn's criteria have been discussed by the cosmologist George Ellis (2003; 2007, pp. 1242-1245), who points out that although they are all desirable they are not of equal relevance and may even lead to conflicts, that is, to opposing conclusions with regard to theory choice. Still, Ellis (and most other cosmologists) finds the first of Kuhn's criteria to be the one that in particular characterizes a scientific theory and demarcates it from other theories. Empirical testability is more than just one criterion out of many.

In cosmology and other areas of fundamental physics it has been agreed for more than a century that both when it comes to theory construction (the context of discovery) and theory evaluation (the context of justification) considerations of an empirical-inductive kind must enter together with hypothetical-mathematical considerations in some proper balance that depends on the case in question. It is also agreed that the empirical elements need not be very important, or can be wholly absent, in the creative or constructive phase of a scientific theory.

Let me illustrate this consensus view with an address that the eminent American physical chemist and mainstream cosmologist Richard Tolman gave in 1932, shortly after the expanding universe had become generally known. In this address, given to the Philosophical Club at the University of California, Los Angeles, Tolman distinguished between two ways of constructing cosmological models, one guided by observational data and the other - paraphrasing Einstein - based on "desiderata for the inner harmony and simplicity of the theoretical structure the physicist is attempting to build" (Tolman, 1932, p. 373). Realizing 
that Einstein had found the field equations of general relativity by the second method, Tolman was nonetheless careful to delimit the purely mathematical considerations to the construction of theories. The physical principles underlying a cosmological theory "must of course in any case agree with observational facts," and even those principles obtained "from the inner workings of the mind" must have consequences that can be presented "to the arbitrament of experimental test."

This was also Einstein's view, even as he moved from a cautious empiricist position inspired by Ernst Mach to an almost full-blown rationalism. In his Herbert Spencer lecture of 1933 he famously stated that "we can discover by means of pure mathematical considerations the concepts and the laws ..., which furnish they key to the understanding of natural phenomena. ... In a certain sense, therefore, I hold it true that pure thought can grasp reality, as the ancients dreamed" (Einstein, 1982, p. 274; Norton, 2000). But in between these two expressions of his rationalist credo, there was the no less important sentence: "Experience remains, of course, the sole criterion of the physical utility of a mathematical construction." As late as 1950, commenting on his new generalized theory of gravitation, he readily admitted that "Experience alone can decide on truth" (Einstein, 1950, p. 17). As we shall see, similar rhetoric is common among modern cosmologists and shared even by many advocates of the existence of numerous unobservable worlds.

\section{Examples from the past}

The modern situation in multiverse cosmology is of great interest from a methodological point of view, but it is not quite unique in the history of cosmological thought. Attempts to introduce major epistemic shifts can be found earlier, both in cosmology and in other parts of the physical sciences (Kragh, 
2011). I shall briefly discuss a couple of episodes from the twentieth century in which epistemic shifts were on the agenda and in which a few physicists suggested to change the "rules of science" in such a way that the first of Kuhn's criteria was essentially disregarded or given very little significance. ${ }^{2}$

The ambitious project of reconstructing fundamental physics that Arthur Eddington pursued between 1929 and his death in 1944 was not a cosmological theory as ordinarily understood, but an attempt to unify quantum mechanics and cosmology under a single mathematical and epistemological framework (Eddington, 1936; 1944; Kilmister, 1994). It was meant to be a theory of everything in the physical universe. The goal of the British astronomer was none other than to deduce all laws and phenomena of nature from epistemological considerations alone, meaning that empirical facts were in principle irrelevant: the laws of nature corresponded to a priori knowledge. In the introduction to his main work Relativity Theory of Protons and Electrons (Eddington, 1936, pp. 3-5), he expressed his apriorism as follows:

All that we require from observation is evidence of identification - that the entities denoted by certain symbols in the mathematics are those which the experimental physicist recognizes under the names "proton" and "electron." Being satisfied on this point, it should be possible to judge whether the mathematical treatment and solutions are correct, without turning up the answer in the book of nature. My task is to show that our theoretical resources are sufficient and our methods powerful enough to calculate the constants exactly - so that the observational test will be the same kind of perfunctory verification that we apply sometimes to theorems in geometry.... I think it will be found that the theory is purely deductive, being based on epistemological principles and not on physical hypotheses.

2 Much has been written about the world systems of Eddington and Milne. References to the literature, both primary and secondary, can be found in Kragh (2011). For the steady state theory, see Balashov (1994) and Kragh (1996). 
Despite its a priori nature, Eddington's theory was rich in empirical consequences, resulting in a large number of precise and apparently testable predictions (or, in most cases, postdictions). To mention just a few, he claimed to have deduced the numerical values of the fine-structure constant $2 \pi e^{2} / h c$, the proton-electron mass ratio $M / m$, the cosmological constant $\Lambda$, and Hubble's recession constant $H_{0}$. However, when the predictions differed from the measured values, he consistently chose to put the blame on the measurements rather than the theory. Indeed he had to, for his unified theory was not testable in any real sense. Nor did Eddington consider ordinary testability to be of much relevance to his theory of the external world, where empirical facts were seen as the necessary consequences of the human mind and not existing in any objective sense. The laws and constants that he deduced were true by necessity and thus could not conceivably be violated by experiments. For example, he maintained that the finiteness and constancy of the speed of light $c$ were given a priori and that measurements of $c$ were therefore, in principle, a waste of time (Eddington, 1946, p. 8). ${ }^{3}$ No wonder that his theory, although not without influence, was dismissed by the large majority of physicists, who either ignored it or severely criticized it on methodological grounds.

At about the same time as Eddington developed his fundamental theory, his colleague in Oxford, the astrophysicist and cosmologist E. Arthur Milne, constructed an alternative "world physics" that shared some of the rationalistic and aprioristic elements of Eddington's system. Although their cosmological views were very different, the two world systems had in common that they were nearly all-encompassing and, on the methodological level, gave high priority to

\footnotetext{
${ }^{3}$ With the spectroscopic redefinition of the metre in 1983 followed a definition of the speed of light in vacuum to be exactly $c=299792458 \mathrm{~m} / \mathrm{s}$ (with uncertainty $\Delta c=0$ ). Since then it has indeed been a waste of time to measure $c$, but only because $c$ is fixed by definition and not for the reasons argued by Eddington.
} 
mathematical reasoning and correspondingly low priority to empirical facts. Much like Eddington, Milne (1948, pp. 10-12) claimed that ultimately the laws of physics could be established from pure reasoning and would then turn out to have the character of mathematical theorems:

Just as the mathematician never needs to ask whether a constructed geometry is true, so there is no need to ask whether our kinematical and dynamical theorems are true. It is sufficient that the structure is self-consistent and free from contradictions; these are in fact the only criteria applied to a modern algebra or geometry. ... Physicists are not accustomed to reasoning about abstract ideas of things; the empirical is always intruding itself, openly or tacitly.

Milne spoke of the "intellectual climate" characterizing his own theory as "markedly different from that ... of current mathematical physics," as indeed it was.

The methodology behind Milne's world physics is not easily described, nor is it perhaps entirely consistent (Cohen, 1950). What matters in the present context is only that Milne, although far from unconcerned with observations, thought that they just played an auxiliary role in verifying results obtained by pure reasoning. His system of world physics could not be falsified by experiment or observation. In an important monograph of 1935 he pointed out that observation can never tell us whether the universe contains an infinite number of objects. Nonetheless, he was convinced that this was actually the case and that it followed unambiguously from his theory. "The philosopher," he wrote, "may take comfort from the fact that, in spite of the much vaunted sway and dominance of pure observation and experiment in ordinary physics, worldphysics propounds questions of an objective, non-metaphysical character which cannot be answered by observation but must be answered, if at all, by pure reason" (Milne, 1935, p. 266). 
Milne's ambitious and unconventional attempt to reform physics from the bottom up was much discussed for a decade or more. Arthur G. Walker - of Robertson-Walker metric fame - was among those who were inspired by Milne's system of what was sometimes known as kinematic relativity. But of course the system was also controversial and occasionally it was accused of transcending the line separating science from non-science. Among the criteria of science adopted by the Cambridge geophysicist Harold Jeffreys (1937) were that a scientific theory must not treat any hypothesis as a priori certain, and that alone was enough to rule out the theories of Milne and Eddington. According to George McVittie, an astronomer and cosmologist of the empirical school, Milne's world physics was essentially to be classified as pseudoscience because it did not deal with nature as observed or as revealed to us experimentally. In a critical review of Milne's and Walker's exposition of kinematic relativity, he fired this broadside: "It is eventually borne in on the puzzled reader that Milne and Walker are not trying to understand Nature but rather are telling Nature what she ought to be. If Nature is recalcitrant and refuses to fall in with their pattern so much the worse for her" (McVittie, 1940, p. 281).

The later controversy over the steady state theory of the universe, pioneered by Fred Hoyle, Hermann Bondi and Thomas Gold in 1948, had some elements in common with the methodological dispute of the 1930s. First and foremost, the steady state theory was sometimes criticized for resting on a priori assumptions such as the perfect cosmological principle, stating that the universe is homogeneous in both space and time. This was in fact an unfair accusation, witness that in the 1960s the perfect cosmological principle was proved wrong by observations - something which is not possible for an a priori principle. Having dealt with this controversy elsewhere (Kragh, 1996), here I just want to point out that Bondi, in particular, justified the new theory methodologically by explicitly 
appealing to its high degree of falsifiability in Popper's sense. Bondi (1992) eventually came to the conclusion that, "There is no more to science than its method, and there is no more to its method than Popper has said." The theory did indeed lead to several sharp and novel predictions of such a kind that, should they turn out to be wrong, the theory must fall. But to critics of the steady state theory this was far from reason enough to prefer it over the standard cosmological theory based on general relativity. As McVittie had criticized Milne for substituting logic for observation, he now complained that the Hoyle-BondiGold theory was essentially rationalistic and lacked an empirical basis. Among its problematic features was that it followed "the dictum that what is logically possible is also physically possible" (McVittie, 1961, p. 1236).

What caused critics such as McVittie, Herbert Dingle, and Mario Bunge to brand the steady state theory as unscientific was in particular the continual creation of matter that was an integral part of the theory and necessary for the perfect cosmological principle to hold in an expanding universe. In an interview of 1978, several years after the original steady state theory had been abandoned, McVittie said about the theory's use of matter creation: "It's like breaking the rules when you are playing a game. If you allow yourself in the game of American football to take knives on board with you and stab your opponents, now and again, of course the results will be very remarkable, particularly if one side only has the knives and the other is merely the recipient" (Kragh, 1996, p. 249). Clearly, cosmologists in the past were concerned about the rules of science that might legitimately be applied to the study of the universe. They still are. Thus, Lee Smolin (2008, p. 170) deplores that some advocates of the multiverse want to "change the rules so that their theory will not need to pass the usual tests we impose on scientific ideas." 


\section{The anthropic multiverse: a brief history}

Disregarding the complex history of early ideas of many universes (Kragh, 2009; Trimble 2009) one may point to several, largely independent origins of the modern concept of the multiverse:

1. The many-worlds interpretation of quantum mechanics, dating back to Hugh Everett's idea of 1957 but only commonly known after it was presented in a modified and more popular form by Bryce DeWitt in 1970.

2. The anthropic principle, as formulated by Brandon Carter in an address of 1973 in which he argued that the weak anthropic principle only had explanatory power if associated with the idea of an ensemble of worlds with different combinations of initial conditions and fundamental constants.

3. The eternal and chaotic inflationary scenarios first proposed in 1982-1983 by Andrei Linde and Alex Vilenkin in the wake of the original inflation hypothesis of Alexei Starobinski and Alan Guth.

4. Mathematical developments in string theory made by Raphael Bousso, Joseph Polchinski and others around 2000. This work indicated that there might be no unique way to compactify the six extra dimensions of the 10-dimensional space-time of string theory and thus produce a unique vacuum state describing the one and only universe.

These four lines of ideas were not initially seen as pointing in the same direction or having much, if anything, in common. For example, it was (and still is) unclear whether the universes of many-worlds quantum mechanics should be thought of as real universes of the same kind as the universe studied by astronomers.

Whereas the anthropic principle made contact to the many-worlds interpretation at an early date - Carter did so in his 1973 address - it was not originally seen as connected to either inflation or string theory. On the contrary, it 
seemed that inflation theory made anthropic explanations redundant or at least limited their domain of application. Why appeal to the anthropic principle to explain the flatness of space, if it followed from the inflationary scenario? Similarly, string theorists had no sympathy for anthropic ideas, which represented an approach to physics quite opposite to the one of string theory (Schellekens, 2008). This theory, if successful, would lead to a unique set of laws and constants of low-energy physics and therefore make the anthropic principle and similar appeals to fine tuning irrelevant (Kane et al., 2002). The situation gradually changed in the 1990s, when some physicists began to think of eternal inflation as naturally connected to the anthropic principle. While Guth originally disliked the anthropic principle, by the late 1990s he found it to be justified as an interesting hypothesis by the multiverse generated by eternal inflation (Guth, 1997).

If the modern multiverse is to be assigned a father, a candidate for paternity may be Linde, who in 1982 related the inflation bubble-universes to the weak anthropic principle. Four years later he made a similar connection to string theory (Linde, 1986, p. 399):

All types of mini-universes in which inflation is possible should be produced during the expansion of the universe ... From this point of view an enormously large number of possible types of compactification which exist, e.g. in the theory of superstrings should be considered not as a difficulty but as a virtue of these theories, since it increases the probability of the existence of mini-universes in which life of our type may appear. The old question why our universe is the only possible one is now replaced by the question in which theories the existence of mini-universes of our type is possible.

The multiverse only became a hot issue in the early years of the new millennium, with the emergence and promotion of the "landscape" version of string theory (Susskind, 2006). According to this version, there is a huge number of vacuum 
states or possible compactifications, each of them corresponding in the lowenergy regime to a distinct universe with its own laws and constants of physics. Moreover, these universes are more than mere mathematical solutions to the string equations. They are held to be no less real than the universe we observe the landscape is populated. However, multiverse physicists rarely explicate what they mean by existence. For some it is apparently enough that the other universes are objects consistent with the basic laws of physics, and they then implicitly rely on the magic of the principle of plenitude. From this point of view it is irrelevant whether they can actually be detected in the normal, empirical sense (e.g., Susskind, 2006, p. 177).

The string landscape was offered as theoretical evidence for the multiverse, and it provided the associated anthropic principle with a much needed support from fundamental physics. What a few years earlier had appeared to be an antagonistic relationship between anthropic reasoning and string physics, now changed into an alliance. Brian Greene experienced a "sort of transformation" which made him "very warm to this possibility of there being many universes, and that we are in the one where we can survive," and he was not the only convert (Ananthaswamy, 2009; Siegfried, 2006; Carr, 2007).

There are different kinds of multiverses and different ways to classify them (Tegmark 2007). The simplest spatial multiverse is uncontroversial, as it is realized in many models of the ordinary Friedmann type. Eddington (1931) pointed out at an early date that the accelerated expansion of a universe with a positive cosmological constant (the Lemaittre-Eddington model) will eventually lead to "a number of disconnected universes no longer bearing any physical relation to one another." However, these causally disjoint subuniverses have evolved from the same universe and they share the same cosmic space-time and laws of physics. More interesting are the bubble universes generated by eternal 
inflation, where there are many big bangs and the universes have different laws of low-energy physics, different coupling constants and particle content, and perhaps even different dimensionality. Although not accessible to us, they are nonetheless genetically connected by, presumably, having a common causal origin in a primary big bang. Ellis (2007) has proposed to call universes of this type a "multi-domain universe."

In addition to the two mentioned types of multiverse there are more radical ideas according to which the multiverse is made up of completely separate universes with no regularity in their properties at all. It seems to be generally accepted that the latter kind of multiverse, advocated by a few physicists, cannot be subject to scientific analysis and must be characterized as an interesting philosophical speculation (Ellis et al., 2004). The same is the case with the mathematical multiverse hypothesis proposed by Max Tegmark (2008). A scientific formulation of these types of multiverse requires some kind of metalaw valid for the entire multiverse, and it is hard to see how to justify such a meta-law.

\section{Testability, falsifiability, and the universe}

It is outside the scope of this paper to analyze the many scientific and philosophical aspects of multiverse physics (Carr, 2006; Ellis et al., 2004; Kragh, 2011), but I want to take up the thread from Section 1 and comment on testability as a necessary epistemic standard for cosmological theories. All (or, bearing Eddington and Milne in mind, nearly all) physicists agree that testability is an epistemic value of crucial importance. They consider it an indispensable precondition for a theory being scientific: a theory which is cut off from confrontation with empirical data just does not belong to the realm of science. 
Testability may admittedly not be relevant to all aspects or in all phases of the development of a theory, but ultimately it cannot be ignored. Multiverse and string theorists are no exception to this rhetorical consensus which finds expression time and again in the literature. But one thing is rhetoric, another is scientific practice and the interpretation of the concept of testability. Here is a brief list of questions that physicists and cosmologists do not agree upon:

1. Actual testability (with present instrument technologies or those of a foreseeable future) is obviously preferable. But should it be required that a theory is actually testable, or will testability in principle suffice?

2. Should a theory result in precise and directly testable predictions, or will indirect testability do? For example, if a fundamental theory $T$ results in several successfully confirmed predictions $P_{1}, P_{2}, \ldots, P_{\mathrm{n}}$, can prediction $P_{\mathrm{n}+1}$ be considered to have passed a test even if it is not actually tested? ${ }^{4}$

3. If a theory does not result in precise predictions, but only in probability distributions, is it then testable? If we can calculate from multiverse or anthropic assumptions at a specified confidence level the probability that we should observe a physical property, such as the cosmological constant, to lie in a certain range, does that mean that the corresponding theory is testable?

\footnotetext{
${ }^{4}$ See, for example, Tegmark (2008, p. 124), who argues that there are reasons to believe in untestable predictions if they follow from a well-established theory with empirical success. On this account the existence of other universes is "tested" by the successfully tested background theories, in this case quantum mechanics and inflation theory. For some believers in the many-worlds interpretation, inflation theory is more or less redundant, since (they claim) the many universes follow unambiguously as a prediction from quantum mechanics. If the many-worlds interpretation is admitted as "the only logical interpretation of quantum mechanics," one also has to admit that "the discovery of quantum mechanics was in fact the discovery that gave a solid scientific basis to [the multiverse]" (Mukhanov, 2007). On a different note, string theorists sometimes suggest that the theory of superstrings has passed an empirical test because it includes gravitation without being designed to do so.
} 
4. Will a real test have to be empirical, by comparing consequences of the theory with experiments or observations, or do mathematical consistency checks also count as sufficient (theoretical) tests?

5. Another kind of non-empirical testing is by way of thought experiments or arguments of the reductio ad absurdum type that played an important role in the controversy over the steady state theory. A cosmological model may lead to consequences that are either contradictory or unacceptably bizarre. How should such arguments enter the overall evaluation picture?

6. At what time in the development of a theory or research programme can one reasonably demand testability? Even if a theory is not presently testable, perhaps it will be so in a future version, such as there are many examples of in the history of science.

7. How should (lack of) testability be weighed in relation to (lack of) other epistemic desiderata? E.g., is an easily testable theory with a poor explanatory record always to be preferred over a non-testable theory with great explanatory power? Or what if the testable theory is overly complicated, and the non-testable one is mathematically unique and a paragon of simplicity?

To this list may be added the often discussed question of whether or not predictions of novel phenomena should be counted as more important than preor postdictions of already known phenomena. This is a question on which philosophers are divided and where the historical evidence is ambiguous (see, e.g., Schindler 2008).

While most philosophers consider testability to imply not only falsifiability, but also verifiability and other forms of theory assessment based on empirical evidence, according to Popper (1963, p. 36), “Every genuine test of a theory is an attempt to falsify it, or refute it. Testability is falsifiability." Whatever 
the validity of this equation, Popper's falsificationist philosophy has been very influential in a broad range of sciences (Mulkay and Gilbert, 1981). ${ }^{5}$ According to the Australian philosopher and critic of Popper, David Stove (1999, p. 8), "if you scratch a scientist of middle age or older, you are almost certain to meet with a philosophy of science which consists of half-remembered scraps of Popperism." The importance of falsificationist philosophy à la Popper is perhaps particularly visible in the fields of astronomy, astrophysics and cosmology. As mentioned in Section 2, it played an important role in connection with the steady state theory in the 1950s, and scientists (more than philosophers) continue to take it seriously. Incidentally, so did Einstein, who was very pleased with Logik der Forschung, which he read soon after it appeared in the fall of 1934.6 Popper $(1959$, p. 313) first stated his famous demarcation criterion in a letter to the journal Erkenntnis in 1933.

As shown by Benjamin Sovacool (2005), astronomers and cosmologists often invoke Popper's ideas as a guide for constructing and evaluating theories, although they rarely reveal a deeper familiarity with these ideas. In a review of the state of cosmology at the millennium, the astrophysicist Michael Turner (2001, p. 656) advocated the new standard ( $\Lambda$ CDM) cosmology including inflation and cold dark matter by relating it to Popper's views: "However, with its unidentified dark matter and mysterious dark energy, it is currently very much out on a limb. According to Karl Popper that's what strong theories do! ... Inflation + cold dark matter is bold and testable." Some years later, Smolin (2007,

\footnotetext{
${ }^{5}$ For a full account of Popper's view of and influence on cosmology, see Kragh (2012a). A revised version of this paper has been accepted for publication in Perspectives on Science.

6 "Your book has pleased me very much in many ways: rejection of the 'inductive method' from an epistemological standpoint. Also falsifiability as the crucial element of a theory (of reality) ... . You have further defended your positions really well and astutely." Einstein to Popper, 15 June 1935, as quoted in Van Dongen (2010, p.43).
} 
p. 34) argued that Popper's falsifiability criterion is so basic to science that it amounts to an "ethical imperative." To his mind, it follows from this imperative that the anthropic multiverse is excluded from the domain of science.

It is an important feature of Popper's philosophy that a scientific theory must set constraints to the results of observations. "Every 'good' scientific theory is a prohibition: it forbids certain things to happen," Popper (1963, p. 36) says. "The more a theory forbids, the better it is." But multiverse cosmology - where, in some versions, all that can happen, happens - seems to be anything but prohibitive. As Ellis points out, the landscape multiverse allows virtually any physical state of affairs: “The existence of universes with giraffes is certainly predicted by many multiverse proposals, but universes where giraffes do not exist are also predicted. Observing a giraffe neither confirms nor disproves the multiverse" (Carr and Ellis, 2008, p. 2.35). The authority of Popper's view among cosmologists is further illustrated by the fact that it is invoked, if not without reservations, even by some scientists in favour of the multiverse hypothesis. One of them, the French cosmologist Aurélien Barrau (2007), says:

Except in some favourable cases - for example when all the universes of the multiverse present a given characteristic that is incompatible with our universe - it is hard to refute explicitly a model in the universe. But ... the multiverse remains within the realm of Popperian science. It is not qualitatively different from other proposals associated with usual ways of doing physics. ... Moreover, falsifiability is just one criterion among many possible ones and it should probably not be over-determined.

Stephen Hawking is another theoretical physicist in favour of the multiverse. Much like Einstein, he praises the power of mathematical elegance and logical self-consistency in theory construction. On the other hand, when a theory has been constructed by means of such considerations, "The theory then makes predictions, which can then be tested by observation." Moreover: "If the 
observations agree with the predictions, that doesn't prove the theory; but the theory survives to make further predictions which again are tested against observations. If the observations don't agree with the predictions, one abandons the theory" (Hawking, 1994, p. 36). It is unlikely that Popper would have objected to this formulation. ${ }^{7}$

Sceptics such as Ellis and Smolin are more inclined to consider the multiverse qualitatively different from ordinary physics, perhaps implying an epistemic shift. They do not agree that it remains within the realm of Popperian science. Neither does the leading multiverse physicist Leonard Susskind, one of the fathers of the string landscape, except that for him this is a virtue rather than a problem. Why should scientists respect the standards associated with Popper's critical rationalism and promoted by the modern "popperazi"? Susskind (2006, pp. 193-195) does not:

Throughout my long experience as a scientist I have heard unfalsifiability hurled at so many important ideas that I am inclined to think that no idea can have great merit unless it has drawn this criticism. ... Good scientific methodology is not an abstract set of rules dictated by philosophers. It is conditioned by, and determined by, the science itself and the scientists who create the science. ... Let's not put the cart before the horse. Science is the horse that pulls the cart of philosophy.... Falsification, in my opinion, is a red herring, but confirmation is another story.

What Susskind calls confirmation or testing includes mathematical consistency, a method of testing that "should not be underestimated." Schellekens (2008) agrees

\footnotetext{
7 But Popper's philosophy is normative. Hawking adds: "Or rather, that is what is supposed to happen. In practice, people are very reluctant to give up a theory in which they have invested a lot of time and effort. They usually start by questioning the accuracy of the observations. If that fails, they try to modify the theory in an ad hoc manner." Hawking's observation is a rather precise description of how Hoyle reacted to the observational problems into which the steady state theory ran in the 1960s.
} 
that for the string multiverse "consistency may be the only guiding principle we have," adding that fortunately this is a most powerful principle.

Physicists' and cosmologists' discussions of the merits and faults of Popper's philosophy of science are often overly simplistic and based on misunderstandings (the same was the case with the earlier discussion related to the steady state theory of the universe). What they discuss is most often versions of naïve falsificationism rather than its sophisticated versions (Heller, 2009, pp. 88-89). For example, although Popperian methodology rules out a theory if it cannot be falsified even in principle, it does not require instant falsifiability. The lack of testable implications may not be a permanent feature, in which case the theory will be considered a legitimate research project that may well lead to a truly scientific theory, such as happened with metaphysical atomism at the time of Dalton (Popper, 1974b, p. 981). Popper was well aware that refutation is itself fallible and that it may be rational to keep even an admittedly false theory alive for some time. Adopting a conventionalist position he even suggested that "the testing of a theory depends upon basic statements whose acceptance or rejection, in its turn, depend on our decisions" (Popper, 1959, p. 108). Moreover, Popper never held that falsifiability is a sufficient condition for a theory being scientific, only that it is a necessary condition. There are obviously falsifiable statements that do not qualify as scientific. After all, creationist theories that claim the age of the Earth to be 6,000 years are eminently falsifiable yet patently non-scientific.

There are undoubtedly good reasons for criticizing parts of Popper's view of science, such as many philosophers have done since the publication of The Logic of Scientific Discovery more than half a century ago. While Popperian falsificationism may have agreed with Einsteinian physics in the early decades of the twentieth century, and with the theory of general relativity in particular, in other cases it leads to the absurd conclusion that some of the great theories in the 
history of science were unscientific. Certainly, Popper's philosophy of science bears little resemblance to what most working scientists do today, which has led to the suggestion that falsificationism has itself been falsified or is no longer a relevant demarcation criterion (Hansson, 2006).

Like in cosmology, within some fields of the biological sciences Popperian standards are assumed to be authoritative in assessing the scientific status of theories. Biologists do not necessarily follow these standards, but they generally find them attractive (Rieppel, 2008). On the other hand, and also like in cosmology, there are biologists who rather conclude that if their science does not live up to Popper's philosophical views, so much the worse for these views. Popper's claim that Darwinism was not really a testable scientific theory but a metaphysical research programme, was not well received by evolutionary biologists (Popper, 1974a, p. 134; Yang, 2008). Although Popper soon reversed his position and admitted that Darwinian evolution theory was indeed scientific, the incident caused some biologists to doubt the epistemic values of falsifiability and strict testability. In a paper of 2005 two biologists advocated that their colleagues should stop paying attention to the work of philosophers, whether Popper or others. Their conclusion, rhetoric and choice of metaphor were strikingly similar to those used by Susskind at the same time in the very different field of cosmology: "To date, philosophy has been the horse pulling the science cart ... It might be timely to allow science to play the part of the horse pulling the cart of philosophy" (Helfenbein and DeSalle, 2005, p. 279; see also Yang, 2008, who explicitly compares the situation in biology with the one in multiverse cosmology). 


\section{Popper on cosmology}

Although of no direct relevance to this paper, it may be useful to point out that whereas Popper's philosophy of science has been highly influential in the development of cosmology, the reverse is not the case: physical cosmology seems to have been largely unimportant to the emergence and evolution of Popper's ideas. It was a subject he did not deal with in any of his major publications, and yet it is not entirely absent from his work. An exception is a little known paper of 1940 in which he, inspired by Milne's methods, proposed to interpret the galactic redshifts within a conventionalist perspective (Popper, 1940). Although in Logik der Forschung he declared that "All science is cosmology," with the term "cosmology" he did not usually refer to attempts to understand the universe on the basis of the laws of physics. He mostly used the term in the much broader sense of natural philosophy, as "understanding the world - including ourselves, and our knowledge, as part of the world" (Popper, 1959, p. 15).

In Objective Knowledge, Popper (1972, p. 186, based on a lecture of 1968) briefly commented on modern cosmological hypotheses, which he considered "the most interesting kind of all hypotheses." Some of these, he said, "can of course be tested, and some have been even sufficiently precise for refutation. But others, and very interesting ones, seem to be untestable, and may remain so." Most likely he referred to the steady state theory and the new big bang theory, respectively. His most explicit commentary on modern cosmology appeared in a lecture given in 1982 at the European Alpbach Forum in Alpbach, Austria. According to Popper (1994, pp. 58-60), the Hoyle-Bondi-Gold steady state theory - "in my view, a very fine and promising theory" - was not only testable but refuted by radio-astronomical measurements in favour of the big bang theory. Without speaking out against the big bang theory, he remarked that "we seem to be almost as helpless in the field of cosmology in the face of some of these 
revolutionary results as we are in politics when faced with the task of making peace." While originally admiring the relativistic big bang theory, he came to see it as nearly irrefutable and based on illegitimate hypotheses such as the cosmological uniformity principle. Even after the emergence of the standard model in the late 1960s, he remained opposed to the theory and preferred a kind of modified steady state theory that avoided the problem of a finite past (Kragh, 2012a; Kragh, 2012b).

\section{Whose criteria of science?}

The current discussion concerning the multiverse is of obvious interest to the philosophy of science. It involves two major and to some extent related questions which may be stated as follows. (i) Has cosmology become truly and exclusively scientific, in the sense, accepted by many physicists, that philosophical considerations no longer play a legitimate role? If so, has it achieved this remarkable status by changing the rules of science? (ii) Which people or groups have the "right" to define these rules of science and thus to decide whether or not a particularly theory discussed by the scientists is in fact scientific?

It is evident that physical cosmology has progressed dramatically for nearly a century and made many philosophical views about the universe obsolete. We no longer have to appeal to philosophical arguments to know whether or not the universe as a whole is a state of dynamical evolution. On the other hand, one may doubt if the situation is qualitatively different from the one in the 1950s, when Bondi and Gerald Whitrow discussed the scientific nature of cosmology in the pages of the British Journal for the Philosophy of Science (Kragh, 1996, pp. 233-236). Whereas Bondi held that science had now replaced philosophy in cosmology, according to Whitrow philosophical arguments would always remain an essential part of the scientific study of the universe. 
It is far from clear whether some of the recent developments, such as multiverse cosmology and physical eschatology, belong primarily to science or philosophy. The idea of many universes, traditionally a subject of philosophical speculation, is now claimed to have been appropriated by the physical sciences. Is this yet another conquest of the ever-victorious physics, at the expense of philosophy? "The borderline between physics and philosophy has shifted quite dramatically in the last century," Tegmark comments (Seife, 2004). "Parallel universes are now absorbed by that moving boundary. It's included within physics rather than metaphysics." However, sceptics disagree. As Ellis has insisted in several works (2007; Stoeger et al, 2008), there are important problems in cosmology that can only be answered, if they can be answered at all, on a philosophical basis. One of them is the hypothesis of many universes, another concerns the possibility of an infinite number of objects in the universe, and a third problem of a philosophical or metaphysical nature is the absolute origin of the universe. As a fourth but more dubious problem there is the old conundrum of why something exists rather than nothing (Rundle, 2004; Heller, 2009, pp. 177190).

Some physicists advocating the multiverse and anthropic reasoning have questioned whether there is any need for external norms of science of a philosophical nature, these norms being Popperian or something else. "If scientists need to change the borders of their own field of research," says Barrau (2007), "it would be hard to justify a philosophical prescription preventing them from doing so." Not only does he argue that physics can only be defined by the physicists, he also appeals (somewhat inconsistently) to alternative, nonPopperian demarcation criteria proposed by "sociologists, aestheticians and epistemologists." Interestingly, he compares the modern developments in physics and cosmology with the change that has occurred in the evaluation of the 
arts: "Nearly all artistic innovations of the 20th century have transgressed the definition of art as would have been by a 19th-century aesthetician. Just as with science and scientists, art is internally defined by artists." The comparison seems to be flawed. Not only is science a deeply rational affair, which art is not, it also successfully aims at describing and explaining the external world in objective terms. It is undoubtedly true that much of what is appreciated as great art today would not have counted as art at all in the nineteenth century. But the same is not true for the sciences, at least not to the same extent. Disregarding extreme Kuhnian and Feyerabendian views, the sciences are confined by the laws of nature and progress in a much more cumulative manner.

The quotation from Barrau expresses a general distrust of academic philosophy of science, a wish to ignore what philosophers have to say about the difference between science and non-science. It is up to the scientists, and to them only, to define the borders of science. As is usual in cases of controversy, this is a view that has been contested by other scientists. According to the American physicist Robert Ehrlich (2006, p. 282), “decisions as to what constitutes a legitimate scientific theory are simply too important to be left to the practitioners of that field, who obviously have a vested interest in it, such as a desire to keep the funding coming." The background for Ehrlich's comment was the anthropic string multiverse, but he also had intelligent design and creationist science in mind.

Unsurprisingly, in this question Susskind sides with Barrau, suggesting to forget about normative prescriptions and let the scientists, or perhaps the scientific community, determine by way of their practices what is and what is not science: "It would be very foolish to throw away the right answer on the basis that it doesn't conform to some criteria for what is or isn't science" (Brumfiel, 2006). This is however a problematic argument (Kragh, 2011, p. 365). Not only is 
it circular reasoning to define science as what scientists do, it also presupposes that all scientists have roughly the same ideas of what constitutes science, which is definitely not the case. Not even within relatively small fields such as theoretical cosmology or systematic biology is there any consensus. Subjects that scientists find interesting and discuss at conferences or write articles about in peer-reviewed journals do not automatically belong to the realm of science. Moreover, it makes no sense to speak of a "right answer" without appealing, explicitly or implicitly, to some criteria of science. To conclude that a theory is either valid or invalid necessarily involves certain standards of scientific validity. These standards need not be part of a rigid philosophical system ("dictated by the philosophers"), nor do they have to be explicitly formulated, but it is hard to see how they can be avoided. Nature herself does not provide us with the criteria for when an answer is right.

In any case, if anthropic multiverse physics is accepted as truly scientific, it will constitute an epistemic shift, a major methodological discontinuity in the history of modern physics. "We are in the middle of a remarkable paradigm shift," says A. N. Schellekens (2008, p. 1), referring to the anthropic string landscape. Whether particle physics and cosmology are in fact undergoing a revolution and on their way to a new paradigm, or a new set of epistemic standards, remains to be seen.

\section{Conclusions}

Neither scientists nor philosophers are usually concerned with the question of defining science, scientists because they do not need a definition and philosophers because they do not believe there is one. The question has nevertheless become relevant in the modern debate about Multiverse or Universe?, the title of a book published in 2007 and based on a conference held at Stanford 
University four years earlier (Carr, 2007). At least some versions of the multiverse have come under fire for betraying traditional norms of science, thereby crossing the line between science and non-science. This is not the first time such accusations have been raised against cosmological theories, nor will it be the last, but the present controversy differs in some respects from the earlier ones, such as the rationalistic cosmologies of Eddington and Milne and the steady state theory of Hoyle, Bondi and Gold. It should also be noted that the anthropic multiverse is not the only branch of modern theoretical cosmology that faces problems when confronted with testability and other established criteria of what constitutes science. So-called physical eschatology, a name used for extrapolations of the state of the universe to the far future and the possibility of intelligent life in that future, is another research area that gives a nearly free rein to cosmologists' speculations (Kragh, 2011, pp. 332-349).

The multiverse debate is to a large extent about the relationship between philosophy and physical cosmology, and more specifically about the role of empirical testability in the evaluation of cosmological theories. For reasons that are in part historical, Popper's philosophy has dominated much of the debate, while alternative conceptions of theory choice and development (such as Bayesian approaches and Peircean retroduction) have played a much more limited role. Falsifiability remains the crucial issue. While it is taken very seriously by the majority of physicists and cosmologists, some protagonists of the multiverse claim that even theories of the universe that are, for all practical purposes, beyond falsification, qualify as good science. They are not against testability, but tend to deny that testability implies falsifiability; and they interpret testability more broadly than usual. In effect, they are proposing an epistemic shift in the criteria for what should pass as science. 
Perhaps the multiverse will die a natural death, turn out to be merely a temporary aberration in the history of modern cosmology. But even then the controversy will remain an instructive case for future historians and philosophers of science, a case illustrating the ever uneasy relationship between science and philosophy. It will be a useful reminder that the definition of science, or the demarcation between science and non-science, is not and probably never will be a closed chapter in the history of scientific thought.

\section{Acknowledgment}

I thank Samuel Schindler for helpful discussions and critical reading of a draft version of this paper.

\section{References}

Ananthaswamy, A. (2009). Somewhere over the cosmos. New Scientist, 202 (issue 2706), 35-37.

Balashov, Y. (1994). Uniformitarianism in cosmology: background and philosophical implications of the steady-state theory. Studies in History and Philosophy of Science, 25, 933-958.

Barrau, A. (2007). Physics in the universe. Cern Courier, 20 November.

Brumfiel, G. (2006). Outrageous fortune. Nature, 439, 10-12.

Bondi, H. (1992). The philosopher for science. Nature, 358, 363.

Carr, B. \& Ellis, G. F. R. (2008). Universe or multiverse? Astronomy \& Geophysics, 49, 2.292.37 .

Carr, B., Ed. (2007). Universe or multiverse? Cambridge: Cambridge University Press.

Cohen, R. S. (1950). Epistemology and cosmology: E. A. Milne's theory of relativity. Review of Metaphysics, 3, 385-405.

Eddington, A. S. (1931). The expansion of the universe. Monthly Notices of the Royal Astronomical Society, 91, 412-416.

Eddington, A. S. (1936). Relativity theory of protons and electrons. Cambridge: Cambridge University Press.

Eddington, A. S. (1946). Fundamental theory. Cambridge: Cambridge University Press.

Ehrlich, R. (2006). What makes a theory testable, or is intelligent design less scientific than string theory? Physics in Perspective, 8, 83-89. 
Einstein, A. (1950). On the generalized theory of gravitation. Scientific American, 182 (April), 13-17.

Einstein, A. (1982). Ideas and opinions. New York: Three Rivers Press.

Ellis, G. F. R. (2003). The unique nature of cosmology. In A. Ashtekar et al. (Eds.), Revisiting the foundations of relativistic physics (pp. 193-220). Dordrecht: Kluwer Academic.

Ellis, G. F. R. (2007). Issues in the philosophy of cosmology. In J. Butterfield \& J. Earman (Eds.), Philosophy of physics (pp. 1183-1286). Amsterdam: North-Holland.

Ellis, G. F. R., Kirchner, U. \& Stoeger, W. R. (2004). Multiverses and physical cosmology. Monthly Notices of the Royal Astronomical Society, 347, 921-936.

Guth, A. H. (1997). The inflationary universe. Reading, Mass.: Addison-Wesley.

Hansson, S. O. (2006). Falsificationism falsified. Foundations of Science, 11, 275-286.

Hawking, S. (1994). Black holes and baby universes, and other essays. London: Bantham Books.

Helfenbein, K. G. \& DeSalle, R. (2005). Falsification and corroboration: Karl Popper's influence on systematics. Molecular Phylogenetics and Evolution, 35, 271-280.

Heller, M. (2009). Ultimate explanations of the universe. Berlin: Springer-Verlag.

Jeffreys, H. (1937). Physical science and philosophy. Nature, 139, 1004-1005.

Kane, G. L., Perry, M. J. \& Zytkow, A. N. (2002). The beginning of the end of the anthropic principle. New Astronomy, 7, 45-53.

Kilmister, C. W. (1994). Eddington's search for a fundamental theory: a key for the universe. Cambridge: Cambridge University Press.

Kragh, H. (1996). Cosmology and controversy: the historical development of two theories of the universe. Princeton: Princeton University Press.

Kragh, H. (2009). Contemporary history of cosmology and the controversy over the multiverse. Annals of Science, 66, 529-551.

Kragh, H. (2011). Higher speculations: grand theories and failed revolutions in physics and cosmology. Oxford: Oxford University Press.

Kragh, H. (2012a). "The most philosophically important of all the sciences": Karl Popper and physical cosmology. http://philsci-archive.pitt.edu/id/eprint/9062.

Kragh, H. (2012b). Karl Popper on physical cosmologies. Journal for the History of Astronomy, 43, 347-350.

Kuhn, T. S. (1977). The essential tension: selected studies in scientific tradition and change.

Chicago: University of Chicago Press.

Linde, A. (1986). Eternally existing self-reproducing chaotic inflationary universes. Physics Letters B, 175, 395-401.

Matthews, R. (2008). Some swans are grey. New Scientist, 198 (10 May), 44-47.

McVittie, G. C. (1940). Kinematical relativity. The Observatory, 63, 273-281. 
McVittie, G. C. (1961). Rationalism and empiricism in cosmology. Science, 133, 1231-1236. Milne, E. A. (1935). Relativity, gravitation and world-structure. Oxford: Clarendon Press. Milne, E. A. (1948). Kinematic relativity. Oxford: Clarendon Press.

Mukhanov, V. (2007). Cosmology and the many worlds interpretation of quantum mechanics. In B. Carr (Ed.), Universe or multiverse? (pp. 267-274). Cambridge: Cambridge University Press.

Mulkay, M. \& Gilbert, G. N. (1981). Putting philosophy to work: Karl Popper's influence on scientific practice. Philosophy of the Social Sciences, 11, 389-407.

Norton, J. D. (2000). "Nature is the realization of the simplest conceivable mathematical ideas": Einstein and the canon of mathematical simplicity. Studies in History and Philosophy of Modern Physics, 31, 135-170.

Popper, K. R. (1940). Interpretations of nebular red-shifts. Nature, 145, 69-70.

Popper, K. R. (1959). The logic of scientific discovery. New York: Basic Books.

Popper, K. R. (1963). Conjectures and refutations. New York: Routledge.

Popper, K. R. (1972). Objective knowledge: an evolutionary approach. Oxford: Clarendon Press.

Popper, K. R. (1974a). Darwinism as a metaphysical research programme. In P. A. Schilpp (Ed.), The philosophy of Karl Popper (pp. 133-143). LaSalle, Ill.: Open Court. Popper, K. R. (1974b). Replies to my critics. In P. A. Schilpp (Ed.), The philosophy of Karl Popper (pp. 961-1198). LaSalle, Ill.: Open Court.

Popper, K. R. (1994). In search of a better world: lectures and essays from thirty years. London: Routledge.

Rieppel, O. (2008). Popper and systematics. Systematic Biology, 52, 259-271.

Rundle, B. (2004). Why there is something rather than nothing. Oxford: Oxford University Press.

Schellekens, A. N. (2008). The emperor's last clothes? Overlooking the string theory landscape. Reports on Progress in Physics, 71, 072201.

Schindler, S. (2008). Use-novel predictions and Mendeleev's periodic table. Studies in History and Philosophy of Science A, 39, 265-269.

Seife, C. (2004). Physics enters the twilight zone. Science, 305, 464-465.

Siegfried, T. (2006). A "landscape" too far? Science, 313, 750-753.

Smolin, L. (2007). Scientific alternatives to the anthropic principle. In B. Carr (Ed.), Universe or multiverse? (pp. 323-366). Cambridge: Cambridge University Press.

Smolin, L. (2008). The trouble with physics. London: Penguin Books.

Sovacool, B. (2005). Falsification and demarcation in astronomy and cosmology. Bulletin of Science, Technology \& Society, 25, 53-62.

Stoeger, W. R., Ellis, G. F. R., \& Kirchner, U. (2008). Multiverses and cosmology: philosophical issues. arXiv:astro-ph/0407329v2. 
Stove, D. (1999). Against the idols of the age. New Brunswick, NJ: Transaction Publishers. Susskind, L. (2006). The cosmic landscape: string theory and the illusion of intelligent design. New York: Little, Brown and Company.

Tegmark, M. (2007). The multiverse hierarchy. In B. Carr (Ed.), Universe or multiverse? (pp. 99-126). Cambridge: Cambridge University Press.

Tegmark, M. (2008). The mathematical universe. Foundations of Physics, 38, 101-150.

Tolman, R. C. (1932). Models of the physical universe. Science, 75, 367-373.

Trimble, V. (2009). Multiverses of the past. Astronomische Nachrichten, 330, 761-769.

Turner, M. S. (2001). A sober assessment of cosmology at the new millennium.

Publications of the Astronomical Society of the Pacific, 113, 653-657.

Van Dongen, J. (2010). Einstein's unification. Cambridge: Cambridge University Press.

Yang, A. (2008). Matters of demarcation: philosophy, biology, and the evolving fraternity between disciplines. International Studies in the Philosophy of Science, 22, 211-225. 